\title{
Prospective evaluation of kidney displacement during supine mini-percutaneous nephrolithotomy: Incidence, significance, and analysis of predictive factors
}

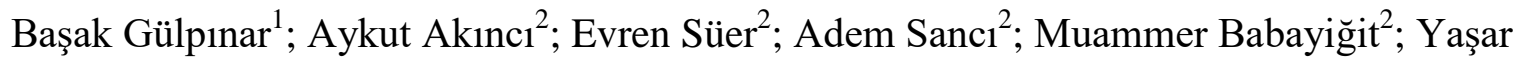
Bedük $^{2}$; Sümer Baltaci²; Mehmet İlker Gökce ${ }^{2}$

${ }^{1}$ Ankara University School of Medicine Department of Radiology; ${ }^{2}$ Ankara University School of Medicine Department of Urology; Ankara, Turkey

Cite as: Can Urol Assoc J 2018 June 8; Epub ahead of print. http://dx.doi.org/10.5489/cuaj.5247

Published online June 8, 2018

\section{$* * *$}

\section{Abstract}

Introduction: Kidney displacement may alter the quality of renal puncture during percutaneous nephrolithotomy (PCNL). The aim of this study was to identify the rate of kidney displacement, and parameters associated with kidney displacement in patients who underwent supine miniPCNL.

Methods: Data of 98 consecutive patients who underwent mini-PCNL was collected prospectively. The patients were grouped as displacement-positive vs. -negative. The parameters collected were age, gender, body mass index, side of the kidney, punctured calyx, fluoroscopy time to successful puncture and tract dilation, stone-free and complication rates, stone diameter, length of the renal artery, quantity of peri-renal and abdominal fat. Groups were compared for the above listed parameters and logistic regression analysis was performed to identify factors associated with kidney displacement.

Results: There were 34 and 64 patients in the displacement-positive and -negative groups, respectively. Groups were similar for stone-free and complication rates. Fluoroscopy time to puncture and tract dilation were longer in the displacement-positive group. Groups were different for renal artery length and peri-renal fat measurements. In multivariate analysis, lower pole puncture, renal artery length, and peri-renal fat measurement were found to be independent predictors of kidney displacement.

Conclusions: Kidney displacement does not alter the success and complication rates, but is associated with longer fluoroscopy times during supine PCNL. In the current study, parameters in preoperative NCCT associated with kidney displacement were identified. We recommend surgeons evaluate and take in to account these parameters during preoperative planning to establish better outcomes and diminish fluoroscopy times. 


\section{Introduction}

Percutaneous nephrolithotomy (PCNL) is the golden standard treatment option for management of stones $>20 \mathrm{~mm}$ in diameter. ${ }^{1,2}$ Although prone position is currently the most commonly preferred position, performing the procedure in the supine position with various modifications has been reported to be equally effective and safe. ${ }^{3-6}$

Supine position has important advantages over prone position. First of all, the total operative times are shorter as patient repositioning is not needed. Also injuries during patient repositioning and cardiopulmonary effects of prone position are avoided. Finally, supine position is more convenient for endoscopy combined intrarenal surgery and, allows easier upper pole access through a lower pole puncture. ${ }^{7-9}$

Access to the collecting system is the most important step in PCNL and an important problem during access is the kidney displacement. Due to the lack of support on the anterior body wall, kidney displacement has been hypothesized to be a problem in modified supine position. The current literature lacks studies evaluating the importance of kidney displacement. In one of the studies, Falahatkar et al. analyzed the factors associated with kidney displacement and identified BMI and prone position as significant factors. However, this study included relatively lower number of patients (33 patients in total) and included only age, gender, patient position, and BMI in the regression analysis. ${ }^{10}$

In the current study, we aimed to identify the rate of kidney displacement, its effect on achievement of a successful puncture, and parameters associated with kidney displacement in a prospective cohort of patients underwent PCNL in modified supine position.

\section{Methods}

In this study the data of 98 consecutive patients that underwent mini-PCNL in our department for renal stones between September 2016 and August 2017 was collected prospectively. Informed consent was obtained from all individual participants included in the study. All of the operations were performed by a single experienced surgeon (MIG) in the Galdakao modified supine Valdivia position. In all cases the tract was dilated to 16 Fr and 12 Fr nephroscope (Karl Storz, Tuttlingen, Germany) was used. Patients underwent PCNL with a greater tract size were excluded due to fact that dilation method and the tract size has significant impact on kidney displacement. Patients with a history of previous percutaneous or open stone surgery were also excluded as this parameter may also result in fixation of the kidney due to fibrosis of the perirenal tissues.

All of the patients underwent a non-contrast enhanced computerized tomography (NCCT) prior to operation as a routine imaging prior to surgery. All examinations were performed with a 64 multi-detector CT (Aquillion 64, Toschiba, Tokyo, Japan). Scan parameters 64 x 0.5 mm collimeters, pitch value 1.484, $5 \mathrm{~mm}$ section thickness, $1 \mathrm{~mm}$ reconstruction interval, $120 \mathrm{kV}$, automatic $\mathrm{mA}$ value and 0.5 seconds rotation time. After axial images were assessed, coronal and sagittal reformatted images and three dimensional (3D) reconstructions were performed with the maximum intensity projection (MIP) and volume rendering methods. Axial thin-section MIP, 
curved coronal reformation across the renal arteries and 3D volume rendered images were used to assess renal arterial anatomy. A single radiologist (BG) performed the measurements on NCCT images and she was blinded to the results of kidney displacement. The measurement from the NCCT were stone diameter (three dimensions), length of the renal artery, quantity of the peri-renal fat, and abdominal fat. Length of the renal artery was measured on the ipsilateral side.

The peri-renal and abdominal fat measurements were performed based on the methodology previously described by Anderson et al. ${ }^{11}$ The peri-renal fat was assessed at three locations. The anterior peri-renal fat (the distance between the nearest overlying bowel where the main renal vein enters the renal hilum or the shortest distance from the anterior renal capsule to Gerota's fascia); the posterior peri-renal fat (the distance between the posterior renal capsule to the nearest posterior abdominal wall at the point where the main renal vein entering the renal hilum) and lateral peri-renal fat (the shortest distance from the inferior tip of liver or spleen to kidney) were these three locations.

Abdominal wall fat was also measured at three sites; including anterior abdominal fat (the distance from linea alba to the skin); lateral abdominal fat (the distance from the lateral abdominal wall muscles to the skin) and posterior abdominal fat (the distance from the tip of the vertebral spinous process to the skin). Abdominal wall fat was all assessed at the same CT level where the peri-renal fat measurements were performed.

The other parameters recorded for the analysis were age, gender, body mass index (BMI), side of the kidney (right or left), stone location (location of the most prominent stone in case of multiple stones), punctured calyx (upper, middle or lower), fluoroscopy time to successful puncture, fluoroscopy time to tract dilation, stone free rate (defined as no residual fragments), and complication rates. Stone volume (SV) was represented in $\mathrm{mm}^{3}$ and was calculated with the formula: $\mathrm{SV}=\pi / 6 \mathrm{X}$ (Anteroposterior $\mathrm{X}$ transverse $\mathrm{X}$ cranio-caudal diameters of the stone in $\mathrm{mm})$. In case of multiple stones, the sum of volumes of all stones were analyzed.

The patients were grouped in to two as displacement positive vs. displacement negative for comparison. In order to define kidney displacement, we used the methodology that was previously reported by Falahatkar et al. ${ }^{10} \mathrm{~A}$ transparent graph paper of $5 \times 5 \mathrm{~mm}$ squares were placed on the fluoroscopy monitor and amount of kidney displacement was measured during needle puncture and tract dilation. A displacement more than $10 \mathrm{~mm}$ in any of the stages was accepted as positive displacement. The cut of value of $10 \mathrm{~mm}$ was accepted as the mean value of kidney displacement was reported as $10.7 \mathrm{~mm}$ during tract dilation in the study by Flahatkar et al. $^{10}$

Postoperative stone free status was evaluated before JJ stent extraction by KUB or ultrasound and NCCT was performed in the presence of a suspicious residual fragment. Stone free was defined as absence of any residual fragments. 


\section{Statistical analysis}

Statistical analysis was performed with SPSS ver. 20.0 (IBM Corp. Released 2011. IBM SPSS Statistics for Windows, Version 20.0. Armonk, NY: IBM Corp.). Patient characteristics were summarized using mean $\pm \mathrm{SD}$ for continuous variables and frequency (percentage) for categorical variables. The Chi-square test was used to compare the categorical variables and Mann-Whitney U test was used to compare the continuous variables. Multivariate logistic regression analysis was performed to identify factors associated with kidney displacement. For statistical significance p value of 0.05 was accepted.

\section{Results}

Data of 98 patients were evaluated and the mean age of the population was $46.8 \pm 5.3$. There were 34 patients in the displacement positive group and 64 patients in the displacement negative group. The two groups were similar for mean age, gender, stone location, BMI, and mean SV. The results of the demographic characteristic are summarized in table 1.

The two groups were significantly different for the operated side and the punctured calyx. In the displacement positive group, 21 of the 34 patients (61.8\%) were operated for right kidney, whereas in the displacement negative group 38 of the 64 patients (59.4\%) were operated for the left kidney ( $\mathrm{p}=0.04)$ (table 1 ). Lower pole puncture was also more frequently performed in the displacement positive group compared to the displacement negative group (94.2\% vs. $75 \%$, $\mathrm{p}=0.04$ ) (table 2).

The measurements from the NCCT studies are summarized in table 3. The two groups were similar for stone size and abdominal fat measurements. However, the renal artery length was significantly longer in the displacement positive group compared to the displacement negative group ( $42.2 \pm 5.1 \mathrm{~mm}$ vs. $36.4 \pm 4.5 \mathrm{~mm}, \mathrm{p}=0.03$ ). Additionally, the mean peri-renal fat measurements in all three measurement sites were significantly lower in the displacement positive group compared to the displacement negative group (table 3).

The factors that showed a significant difference between the two groups in the univariate analysis were involved in a multivariate logistic regression analysis. These parameters were; laterality (right vs. left), punctured calyx (lower calyx vs. other calices), length of the renal artery, and peri-renal fat measurement (average of the anterior, lateral and posterior fat measurements). In the multivariate analysis, lower pole puncture, longer renal artery length, and lower peri-renal fat measurement were found to be independent predictors of kidney displacement. However, laterality was not found be associated with kidney displacement. The results of the multivariate logistic regression analysis are summarized in table 4.

The groups were similar for the stone free rate $(91.2 \%$ vs. $92.2 \%, \mathrm{p}=0.86)$ and complication rates ( $8.8 \%$ vs. $6.3 \%, \mathrm{p}=0.63$ ) (table 2 ). However, mean fluoroscopy time to successful puncture was significantly longer in the displacement positive group (18.8 \pm 5.4 seconds vs. $11.3 \pm 4.1$ seconds, $\mathrm{p}=0.01$ ). Similarly, mean fluoroscopy time to tract dilation was also significantly longer in the displacement positive group (23.6 \pm 5.8 seconds vs. $15.2 \pm 5.1$ seconds, $\mathrm{p}=0.01$ ). The results are summarized in table 2 . Additionally, in one of the patients in 
the displacement positive group puncture to the desired lower pole calyx was not possible due to the extreme mobility and displacement of the kidney. In this patient renal access was established through an upper calyx and flexible ureterorenoscope was employed for laser lithotripsy and fragment extraction and this patient was one of the three patients with a residual fragment in the displacement positive group.

\section{Discussion}

Kidney displacement during PCNL in the supine position is a common condition and it is observed in about $1 / 3$ of the cases in the current study. Although it was not found to be associated with lower success and higher complication rates, significantly longer fluoroscopy times were reported in displacement positive group, which makes this factor a surrogate for more difficult puncture. The current study also identified a longer renal artery, lower peri-renal fat measurement, and puncture through a lower pole calyx as factors associated with kidney displacement.

Anteromedial displacement of the kidney during PCNL in supine position was first reported in 2002 by Shoma et al. The authors compared supine and prone positions and anteromedial displacement was observed in $11 \%$ of the supine cases but in any of the cases operated in prone position. ${ }^{12}$ The rate of kidney displacement in this study was lower compared to our cohort and we believe that this is due to the difference in the definition of displacement.

Falahatkar et al. reported their results in the complete supine position compared with the prone position in 2008. In this study, the authors mentioned that anterior displacement of the kidney was lower in the complete supine position but neither definition of displacement nor data of patients with significant displacement was provided. ${ }^{13}$ The same group reported their comparative data for kidney displacement in complete supine and prone positions in 2011. In this study the authors also attempted to identify factors associated with significant displacement and in order to determine the amount of displacement a graph paper was placed on the fluoroscopic monitor and the kidney movements were recorded. ${ }^{10}$ We also used this methodology in our study and grouped the patients in accordance with cut off levels obtained from the study by Falahatkar et al. ${ }^{10}$

In addition to the position the authors also evaluated gender, age, and BMI as factors that may be associated with displacement. The only parameter that was found to be significant was patient position. Also body mass index was found to be associated with kidney displacement during the tract dilation stages. ${ }^{10}$ In our study we evaluated other parameters and identified puncture of a lower pole calyx, a longer renal artery, and lesser peri-renal fat thickness as factors associated with significant kidney displacement. The BMI and abdominal fat measurements were not found to be significant but, lesser peri-renal fat measurements were significantly associated with kidney displacement. A possible explanation for this finding is peri-renal fat tissue stabilizes the kidney and prevents displacement but abdominal fat, which correlates with BMI, does not have a role. 
In the univariate analysis, right sided kidney was more prevalent in the displacement positive group but it was not found to be a significant factor in the multivariate analysis. We believe that this is associated with the renal artery measurement. The mean length of renal artery was longer in the displacement positive group and hypothetically, renal artery, with its stiff structure, stabilizes the kidney. The length of renal artery is shorter in the left kidney and this explains the lower percentage of left sided kidney in the displacement positive group.

Puncture through a lower calyx was also found to be associated with significant displacement in multivariate analysis. Although we evaluated the displacement in two dimensions under fluoroscopy, the kidney moves in three dimensions. Lower pole puncture results in caudal to cranial movement of the kidney and in some cases the lower pole may rotate from lateral to medial and this makes puncture of the papilla of the desired calyx difficult. For this reason, in one of the cases, puncture of a lower calyx was not possible, and puncture of a middle calyx was performed.

We did not observe any difference in the stone free rates and complication rates in the two groups. Although, kidney displacement was observed in about 1/3 of the cases, it did not have an effect on the final outcomes. However, in the present study all operations were performed by an experienced endourologist (performing >150 PCNL cases per year) and therefore, kidney displacement may have an impact on outcomes of PCNL when performed by an unexperienced surgeon.

The fluoroscopy time to successful puncture and tract dilation were longer in the displacement positive group and we believe that kidney displacement is one of the factors responsible for more difficult percutaneous access. Therefore, during preoperative planning the factors identified as being associated with significant displacement should be kept in mind. For instance, in case of a renal pelvis stone with long renal artery and less amount of peri-renal fat, puncture through a middle calyx instead of a lower calyx may prevent kidney displacement and aid in easier puncture of the collecting system.

The most important limitation of our study is the inclusion of only mini PCNL cases. With the increased tract size, amount of displacement may increase as well. Also there are other factors associated with more difficult puncture and access to the collecting system such as dilation of the target calyx, space around the stone, and abnormal anatomy or malrotation of the kidney. We also excluded patients with history of previous surgery for standardization but this parameter also has potential to be an independent factor. These parameters were not evaluated in the current study. Also there is no universally accepted criteria for significant kidney displacement. To the best of our knowledge there is only one study that objectively measured the amount of displacement. ${ }^{10}$ and we used the data from this study to group the patients. Another important point is kidney displacement has been studied in two dimension fluoroscopic views. However, kidney can move in all three dimensions and we could not evaluate displacement in the anterior-posterior plane. Additionally, presence of residual stones was not evaluated with NCCT in all patients which is gold standard imaging modality. 


\section{Conclusions}

Kidney displacement can occur in up to 1/3 of the PCNL cases operated in supine position. Although, it does not alter the success and complication rates, it is associated with longer fluoroscopy times and therefore more difficult access. Puncture through a lower calyx, longer renal artery, and lesser peri-renal fat measurement were identified as factors associated with significant displacement. These parameters can be evaluated in preoperative NCCT studies and we recommend the surgeons to take in to account these parameters during preoperative planning. 


\section{References}

1. Turk C, Petrik A, Sarica K, et al. EAU Guidelines on Interventional Treatment for Urolithiasis. Eur Urol. 2016;69:475-482.

2. Assimos D, Krambeck A, Miller NL, et al. Surgical Management of Stones: American Urological Association/Endourological Society Guideline, PART I. J Urol. 2016;196:1153-1160.

3. Falahatkar S, Mokhtari G, Teimoori M. An Update on Supine Versus Prone Percutaneous Nephrolithotomy: A Meta-analysis. Urol J. 2016;13:2814-2822.

4. Ibarluzea G, Scoffone CM, Cracco CM, et al. Supine Valdivia and modified lithotomy position for simultaneous anterograde and retrograde endourological access. BJU Int. 2007;100:233-236.

5. Scoffone CM, Cracco CM, Cossu M, Grande S, Poggio M, Scarpa RM. Endoscopic combined intrarenal surgery in Galdakao-modified supine Valdivia position: a new standard for percutaneous nephrolithotomy? Eur Urol. 2008;54:1393-1403.

6. Valdivia JG, Scarpa RM, Duvdevani M, et al. Supine versus prone position during percutaneous nephrolithotomy: a report from the clinical research office of the endourological society percutaneous nephrolithotomy global study. J Endourol. 2011;25:1619-1625.

7. Cracco CM, Scoffone CM. ECIRS (Endoscopic Combined Intrarenal Surgery) in the Galdakao-modified supine Valdivia position: a new life for percutaneous surgery? World J Urol. 2011;29:821-827.

8. Gokce MI, Ibis A, Sanci A, et al. Comparison of supine and prone positions for percutaneous nephrolithotomy in treatment of staghorn stones. Urolithiasis. 2017.

9. Sofer M, Giusti G, Proietti S, et al. Upper Calyx Approachability through a Lower Calyx Access for Prone Versus Supine Percutaneous Nephrolithotomy. J Urol. 2016;195:377382.

10. Falahatkar S, Asgari SA, Nasseh H, et al. Kidney displacement in complete supine PCNL is lower than prone PCNL. Urol Res. 2011;39:159-164.

11. Anderson KM, Lindler TU, Lamberton GR, Baron PW, Ojogho OK, Baldwin DD. Laparoscopic donor nephrectomy: effect of peri-renal fat upon donor operative time. $J$ Endourol. 2008;22:2269-2274.

12. Shoma AM, Eraky I, El-Kenawy MR, El-Kappany HA. Percutaneous nephrolithotomy in the supine position: technical aspects and functional outcome compared with the prone technique. Urology. 2002;60:388-392.

13. Falahatkar S, Moghaddam AA, Salehi M, Nikpour S, Esmaili F, Khaki N. Complete supine percutaneous nephrolithotripsy comparison with the prone standard technique. $J$ Endourol. 2008;22:2513-2517.

Figures and Tables 


\begin{tabular}{|l|l|c|c|}
\hline \multicolumn{4}{|l|}{ Table 1. Comparison of the two groups for the demographic and stone related characteristics } \\
\hline Parameters & $\begin{array}{c}\text { Displacement } \\
\text { positive (n=34) }\end{array}$ & $\begin{array}{c}\text { Displacement } \\
\text { negative (n=64) }\end{array}$ & p \\
\hline Age, mean \pm SD & $46.2 \pm 5.4$ & $47.1 \pm 5.5$ & 0.66 \\
\hline Gender, $n(\%)$ & & & 0.61 \\
\hline Male & $20(58.8)$ & $41(64.1)$ & \\
\hline Female & $14(41.2)$ & $23(35.9)$ & \\
\hline Laterality, n (\%) & & & 0.04 \\
\hline Right & $21(61.8)$ & $26(40.6)$ & \\
\hline Left & $13(38.2)$ & $38(59.4)$ & \\
\hline Stone location, $n(\%)$ & & & 0.99 \\
\hline Upper pole & $1(2.9)$ & $2(3.1)$ & \\
\hline Renal pelvis/middle pole & $18(52.9)$ & $33(51.6)$ & \\
\hline Lower pole & $15(44.2)$ & $29(45.3)$ & \\
\hline Stone volume $\left(\mathrm{mm}^{3}\right)$, mean \pm SD & $1880.8 \pm 765.5$ & $1905.5 \pm 814.7$ & 0.13 \\
\hline Body mass index, mean \pm SD & $27.9 \pm 5.4$ & $28.2 \pm 5.5$ & 0.72 \\
\hline
\end{tabular}

SD: standard deviation.

SD: standard deviation.

\begin{tabular}{|l|c|c|c|}
\hline \multicolumn{4}{|l|}{ Table 2. Operative characteristics and results of the patients } \\
\hline Parameters & $\begin{array}{c}\text { Displacement } \\
\text { positive (n=34) }\end{array}$ & $\begin{array}{c}\text { Displacement } \\
\text { negative (n=64) }\end{array}$ & p \\
\hline Punctured calyx, n (\%) & & & 0.04 \\
\hline Upper & $1(2.9)$ & $2(3.1)$ & \\
\hline Middle & $1(2.9)$ & $14(21.9)$ & \\
\hline Lower & $32(94.2)$ & $48(75)$ & \\
\hline $\begin{array}{l}\text { Fluoroscopy time to successful } \\
\text { puncture(seconds), mean } \pm \text { SD }\end{array}$ & $18.8 \pm 5.4$ & $11.3 \pm 4.1$ & 0.01 \\
\hline $\begin{array}{l}\text { Fluoroscopy time to tract dilation (seconds), } \\
\text { mean } \pm \text { SD }\end{array}$ & $23.6 \pm 5.8$ & $15.2 \pm 5.1$ & 0.01 \\
\hline Stone free rate, n (\%) & $31(91.2)$ & $59(92.2)$ & 0.86 \\
\hline Complication rate, n (\%) & & & 0.63 \\
\hline Grade I & $2(5.9)$ & $3(4.7)$ & \\
\hline Grade II & $1(2.9)$ & $1(1.6)$ & \\
\hline Grade III or higher & - & - & \\
\hline
\end{tabular}




\begin{tabular}{|c|c|c|c|}
\hline Parameters & $\begin{array}{c}\text { Displacement } \\
\text { positive }(n=34)\end{array}$ & $\begin{array}{c}\text { Displacement } \\
\text { negative }(n=64)\end{array}$ & $\mathbf{p}$ \\
\hline Length of renal artery $(\mathrm{mm})$ mean \pm SD & $42.2 \pm 5.1$ & $36.4 \pm 4.5$ & 0.03 \\
\hline \multicolumn{4}{|l|}{ Peri-renal fat $(\mathrm{mm})$ mean $\pm \mathrm{SD}$} \\
\hline Anterior peri-renal fat & $11.2 \pm 2.8$ & $14.1 \pm 3.1$ & 0.04 \\
\hline Lateral peri-renal fat & $10.1 \pm 2.7$ & $12.5 \pm 3.0$ & 0.04 \\
\hline Posterior peri-renal fat & $11.1 \pm 2.9$ & $14.4 \pm 3.7$ & 0.04 \\
\hline \multicolumn{4}{|l|}{ Abdominal fat $(\mathrm{mm})$ mean \pm SD } \\
\hline Anterior abdominal fat & $20.9 \pm 4.4$ & $21.4 \pm 5.1$ & 0.78 \\
\hline Lateral abdominal fat & $16.4 \pm 3.8$ & $16.8 \pm 3.9$ & 0.82 \\
\hline Posterior abdominal fat & $14.1 \pm 2.3$ & $14.4 \pm 3.3$ & 0.87 \\
\hline
\end{tabular}

SD: standard deviation.

\begin{tabular}{|l|c|c|c|}
\hline \multicolumn{4}{|c|}{ Table 4. Results of multivariate logistic regression analysis for kidney displacement } \\
\hline Parameter & OR & $\mathbf{9 5 \%}$ CI & P \\
\hline Laterality (right vs. left), & 1.155 & $0.675-1.343$ & 0.81 \\
\hline Punctured calyx (lower calyx vs. other calices) & 1.776 & $1.003-2.492$ & 0.03 \\
\hline Length of the renal artery & 2.160 & $1.218-5.354$ & 0.007 \\
\hline Peri-renal fat measurement & 2.067 & $1.183-4.708$ & 0.009 \\
\hline
\end{tabular}

CI: confidence interval; OR: odds ratio. 